\title{
The Use of Latent Class Analysis to Estimate the Sensitivities and Specificities of Diagnostic Tests for Squash vein yellowing virus in Cucurbit Species When There Is No Gold Standard
}

\author{
William W. Turechek, Craig G. Webster, Jingyi Duan, Pamela D. Roberts, Chandrasekar S. Kousik, and Scott Adkins
}

First, second, third, and sixth authors: United States Department of Agriculture-Agricultural Research Service (USDA-ARS), U.S. Horticultural Research Laboratory, 2001 South Rock Road, Fort Pierce, FL 34945; fourth author: University of Florida-IFAS, Southwest Florida Research and Education Center, 2685 State Road 29 North, Immokalee 34142; and fifth author: USDA-ARS, U.S. Vegetable Laboratory, 2700 Savannah Highway, Charleston, SC 29414.

Accepted for publication 27 June 2013.

\begin{abstract}
Turechek, W. W., Webster, C. G., Duan, J., Roberts, P. D., Kousik, C. S., and Adkins, S. 2013. The use of latent class analysis to estimate the sensitivities and specificities of diagnostic tests for Squash vein yellowing virus in cucurbit species when there is no gold standard. Phytopathology 103:1243-1251.

Squash vein yellowing virus (SqVYV) is the causal agent of viral watermelon vine decline, one of the most serious diseases in watermelon (Citrullus lanatus L.) production in the southeastern United States. At present, there is not a gold standard diagnostic test for determining the true status of SqVYV infection in plants. Current diagnostic methods for identification of SqVYV-infected plants or tissues are based on the reverse-transcription polymerase chain reaction (RT-PCR), tissue blot nucleic acid hybridization assays (TB), and expression of visual symptoms. A quantitative assessment of the performance of these diagnostic tests is lacking, which may lead to an incorrect interpretation of results. In this study, latent class analysis (LCA) was used to estimate the sensitivities and specificities of RT-PCR, TB, and visual assessment of symptoms as diagnostic tests for SqVYV. The LCA model assumes that the observed diagnostic test responses are linked to an underlying latent (nonobserved) disease status of the population, and can be used to estimate sensitivity

and specificity of the individual tests, as well as to derive an estimate of the incidence of disease when a gold standard test does not exist. LCA can also be expanded to evaluate the effect of factors and was done here to determine whether diagnostic test performances varied among the type of plant tissue being tested (crown versus vine tissue), where plant samples were taken relative to the position of the crown (i.e., distance from the crown), host (i.e., genus), and habitat (field-grown versus greenhousegrown plants). Results showed that RT-PCR had the highest sensitivity (0.94) and specificity (0.98) of the three tests. TB had better sensitivity than symptoms for detection of SqVYV infection (0.70 versus 0.32$)$, while the visual assessment of symptoms was more specific than TB and, thus, a better indicator of noninfection ( 0.98 versus 0.65$)$. With respect to the grouping variables, RT-PCR and TB had better sensitivity but poorer specificity for diagnosing SqVYV infection in crown tissue than it did in vine tissue, whereas symptoms had very poor sensitivity but excellent specificity in both tissues for all cucurbits analyzed in this study. Test performance also varied with habitat and genus but not with distance from the crown. The results given here provide quantitative measurements of test performance for a range of conditions and provide the information needed to interpret test results when tests are used in parallel or serial combination for a diagnosis.
\end{abstract}

Squash vein yellowing virus (SqVYV) is a whitefly (Bemisia tabaci, biotype B)-transmitted ipomovirus within the family Potyviridae. The virus is the causal agent of viral watermelon vine decline, one of the most destructive diseases of watermelon (Citrullus lanatus L.) in the southeastern United States. The virus is distributed widely in southwest and west-central Florida and has also been detected in Indiana, Georgia, and South Carolina $(2,9,35)$ (C. S. Kousik and S. Adkins, unpublished data). Along with watermelon, many cucurbit species are susceptible to the virus $(2,36)$ and several common cucurbit weeds have been identified as reservoirs for SqVYV $(3,4)$. Watermelon vine decline is characterized by a severe and rapid decline of watermelon vines as the crop approaches harvest. Although attached fruit may be mature at the time of decline, many are not marketable because

Corresponding author: W. Turechek;

E-mail address: william.turechek@ars.usda.gov

* The $\boldsymbol{e}$-Xtra logo stands for "electronic extra" and indicates that the online version contains one supplementary file.

http://dx.doi.org/10.1094/PHYTO-03-13-0071-R

This article is in the public domain and not copyrightable. It may be freely reprinted with customary crediting of the source. The American Phytopathological Society, 2013. the fruit on affected plants often exhibit discolored flesh and necrosis within the rind. The potential for sizeable losses was exemplified in the 2003-04 season, when Florida growers lost 50 to $100 \%$ of their crop, costing an estimated U.S. $\$ 60$ to 70 million (19).

Current diagnostic tests for identification of SqVYV-infected plants or plant tissues are based on the reverse-transcription polymerase chain reaction (RT-PCR) $(2,3)$ and tissue blot nucleic acid hybridization assays (TB) (32). In many cases, the first diagnosis, at least on watermelon, is made based on characteristic and distinct visual symptoms of SqVYV infection. However, symptoms such as plant wilting and chlorosis may not be distinct enough for identification for those unfamiliar with the disease. A quantitative assessment of the performance of these diagnostic tests (i.e., RT-PCR, TB, and visual assessment of symptoms) is lacking, which can lead to an incorrect interpretation of test results, particularly when multiple tests have been performed and test results are contradictory. The improper interpretation of test results has a direct impact on conclusions drawn from epidemiological and disease management studies, mandates involving policy or regulatory issues, and, of course, routine diagnosis of suspect plant samples.

To correctly interpret the result of a diagnostic test, the test's sensitivity (the probability of correctly identifying a truly infected 
subject) and specificity (the probability of correctly identifying a truly noninfected subject) must be determined. Sensitivity and specificity can be used to determine a test's predictive value (the probability that a subject testing positive [or negative] is truly infected [or noninfected]), one of the more vital statistics in diagnostic test evaluation (29). To evaluate the performance of a diagnostic test, it is standard to run the test on a set of samples or subjects that have a known infection status. Truly infected subjects are known as cases and truly noninfected subjects are referred to as controls. To establish case and control populations, one generally relies on the results of a "gold standard" diagnostic, which is defined as a procedure (a method or a test) that can be used to indisputably determine the true infection status of a subject. Gold standard procedures, when they exist, generally tend to be time consuming in their implementation (e.g., culturing), expensive (e.g., sequencing), or impractical or unethical to implement (e.g., a subject needs to be destroyed or killed to determine the true disease status), limiting their usefulness in settings where samples need to be processed quickly, large numbers of samples need to be processed, or it is preferable to have subjects survive the testing procedure.

At present, there is not a gold standard for determining the true status of SqVYV infection in plants. At the time of this study, testing of crown tissue via TB was the standard test $(2,32)$. The absence of a gold standard is typical in many diagnostic scenarios, and it can be argued that what is often referred to as a "gold standard" is not a true gold standard $(10,21)$. Accordingly, several methods have been introduced to evaluate diagnostic tests in the absence of a gold standard $(21,34)$. One approach is based on latent class probabilistic models, where the observed diagnostic test responses are linked to the underlying latent (non-

TABLE 1. Number of plants mechanically inoculated (greenhouse) or whitefly inoculated (field) and the number of plants tested for Squash vein yellowing virus (in parentheses) by all three diagnostic tests in the study

\begin{tabular}{|c|c|c|}
\hline Genus and species & Cultivar & $\begin{array}{l}\text { Number of plants } \\
\text { inoculated }\end{array}$ \\
\hline \multicolumn{3}{|l|}{ Greenhouse } \\
\hline Benincasa hispida & Jade & $5(1)$ \\
\hline Citrullus lanatus var. citroides & Citron & $5(5)$ \\
\hline C. lanatus var. lanatus & Crimson Sweet & $5(5)$ \\
\hline \multirow[t]{2}{*}{ Cucumis dipsaceus } & Prickles & $5(1)$ \\
\hline & Hedgehog & $3(3)$ \\
\hline \multirow[t]{5}{*}{ C. melo } & Topmark & $4(1)$ \\
\hline & Jumbo Hales Best & $5(1)$ \\
\hline & Diplomat & $5(5)$ \\
\hline & Lily & $5(1)$ \\
\hline & Honey Yellow & $5(1)$ \\
\hline C. metiliferus & Horned Melon & $4(0)$ \\
\hline C. sativus & Green Long & $5(1)$ \\
\hline \multirow[t]{3}{*}{ Cucurbita maxima } & Blue Ballet & $5(5)$ \\
\hline & Buttercup Green & $5(5)$ \\
\hline & Jarrahdale & $5(5)$ \\
\hline \multirow[t]{2}{*}{ C. moschata } & Waltham & $5(2)$ \\
\hline & La Estrella & $5(2)$ \\
\hline \multirow[t]{2}{*}{ C. реро } & Jack O’ Lantern & $5(1)$ \\
\hline & Baby Boo & $5(2)$ \\
\hline Lagenaria siceraria & Birdhouse & $5(5)$ \\
\hline Momordica charantia & Balsam Apple & $5(5)$ \\
\hline Trichosanthes anguina & Polo & $5(2)$ \\
\hline \multicolumn{3}{|l|}{ Field } \\
\hline \multirow[t]{3}{*}{ Citrullus lanatus var. lanatus } & Crimson Sweet & $10(2)$ \\
\hline & PI392291 & $5(1)$ \\
\hline & PI459074 & $4(1)$ \\
\hline \multirow[t]{2}{*}{ C. lanatus var. citroides } & PI500354 & $5(2)$ \\
\hline & Citron & $10(5)$ \\
\hline \multirow[t]{3}{*}{ Cucurbita maxima } & Blue Ballet & $10(3)$ \\
\hline & Buttercup Green & $10(3)$ \\
\hline & Jarrahdale & $10(3)$ \\
\hline \multirow[t]{2}{*}{ Cucumis dipsaceus } & Prickles & $5(4)$ \\
\hline & Total & $175(83)$ \\
\hline
\end{tabular}

observed) disease status of the population (7). Hui and Walter (20) developed a multinomial model for the two-test, two-population scenario and derived a closed-form maximum likelihood (ML) solution to estimate the sensitivities and specificities of the two tests as well as the disease incidences in the two populations. Their approach has been generalized to account for more than two tests in one or more populations. However, no closed-form ML solutions have been identified for models other than the one introduced by Hui and Walter (20); thus, parameter estimation relies on iterative algorithms to identify ML estimates of test parameters.

The objectives of the research reported here were to (i) evaluate the performance of RT-PCR, TB, and visual assessment of symptoms as diagnostic tests for SqVYV with latent class analysis (LCA) and (ii) determine whether the performance of these tests varies predictably among different cucurbit genera, between cucurbit tissue type (crown versus vine tissue), where samples are taken along the vine relative to the position of the crown (i.e., distance from the crown), and habitat (field- versus greenhousegrown plants). Results of this study are intended to give quantitative measurements of test performance for a range of conditions, and provide the information required to interpret test results when tests are used in parallel or serial combination for a diagnosis.

\section{MATERIALS AND METHODS}

Virus source and inoculum preparation. An isolate of SqVYV collected from watermelon in southwest Florida in 2007 was used in all greenhouse and field inoculations $(2,32)$. The isolate was maintained in both watermelon 'Crimson Sweet' and squash (Cucurbita pepo) 'Prelude II' (Seminis Seeds, Oxnard, CA) as previously described (2). Inoculum was prepared by homogenizing a mixture of infected watermelon and squash foliar tissue in $20 \mathrm{mM}$ sodium phosphate buffer ( $\mathrm{pH}$ 7.0) containing $0.1 \%(\mathrm{wt} / \mathrm{vol})$ sodium sulfite and $1 \%(\mathrm{wt} / \mathrm{vol})$ celite.

Plant production, inoculation, and evaluation. In total, 175 plants were used in this study, with 105 grown and inoculated mechanically in the greenhouse and another 70 grown and inoculated in the field. Cultivars from several genera, including three Citrullus plant introductions (PIs), were chosen to represent the diversity of cucurbit species (including both weedy and cultivated species) known to produce a diverse set of symptoms (Table 1). For greenhouse-grown plants, five plants for each cultivar (except 'Hedgehog' and 'Topmark') were grown upward along strings to conserve space and to produce larger, more manageable plants $(1,36)$. The cotyledons and the six lower-most pairs of true leaves of the greenhouse-grown plants were inoculated mechanically as described by Webster et al. (36) at 50 to 64 days old. Plants were inoculated again at 7 and 14 days after the first inoculation to minimize escapes. Plants were collected for virus testing 28 days after the first inoculation, when plants were 78 to 92 days old, with the exception of 'Crimson Sweet', which was collected after 2 weeks due to the severity in symptom expression.

Plants from the field were collected from two trials conducted at the University of Florida's Southwest Florida Research and Education Center in Immokalee. A full description of the field preparation, experimental design, and planting procedures for both the August 2009 (fall trial) and February 2010 (spring trial) can be found in Webster et al. (36). The PIs and Cucumis dipsaceus 'Prickles' were grown only in the fall trial. A 4-week-old SqVYV-infected squash plant (inoculated mechanically, as described by Webster et al.) (36) was planted at the end of each plot at transplanting to serve as the initial source of inoculum. Plants were assessed visually for disease at a weekly interval beginning approximately 2 weeks after transplanting. The plants were collected for virus testing when they were in an advanced state of decline or 8 weeks after transplanting (whichever came first). Thus, not all plants were collected at the same time. A few plants 
completely collapsed before we were able to harvest and test them.

Sample preparation and diagnostic testing. Plants were prepared for diagnostic testing by cutting approximately $2.5-\mathrm{cm}-\mathrm{long}$ vine segments beginning at the crown of the plant and ending at the vine tip to give eight sampling locations per plant, for a total of 1,400 samples (i.e., $8 \times 175$ plants). Prior to testing, each segment (including fruit peduncles when present on a vine segment) and the crown tissue were individually inspected for symptoms typical of SqVYV infection including necrosis, vein yellowing, petiole collapse for vine tissue, and discoloration of the vascular tissue for crown tissue. Results were recorded. Visual inspection for symptoms was considered a diagnostic test.

TB and RT-PCR are DNA-based diagnostic tests that were used to detect SqVYV in cucurbit samples. For TB, tissue print blots of plants were made from vines of freshly cut cucurbits on positively charged nylon membranes (Roche, Indianapolis, IN). Blots were probed using a digoxigenin (DIG)-labeled cDNA probe of a 1,071-bp region of the SqVYV coat protein (CP). Immobilization, hybridization, and chemiluminescent development of membranes were previously described (32).

For RT-PCR, total RNA was extracted from 100 to $130 \mathrm{mg}$ of tissue using Trizol Reagent (Invitrogen Life Sciences, Carlsbad, CA) using the manufacturer's recommended protocol. Total RNA was suspended in RNase-free water and quantified using a Nanodrop spectrophotometer (Nanodrop Technologies, Wilmington, DE). First-strand cDNA was synthesized using $500 \mathrm{ng}$ of total RNA and a potyvirus $3^{\prime}$ terminus primer (16). A 1,071-bp region of the SqVYV CP gene was amplified by PCR using primers and methods previously described (3). Products were visualized on a $1 \%$ agarose gel stained with ethidium bromide.

LCA. LCA is used to identify a set of discrete, mutually exclusive latent (i.e., unobserved) classes (e.g., healthy and infected) based on the observed responses of individuals or subjects to a set of categorical variables. LCA was introduced by Lazarsfeld (25) and was significantly extended and formalized by Goodman $(17,18)$, who developed the expectation-maximization (EM) algorithm that now forms the basis of many programs that fit LCA models. The algorithm is an iterative algorithm that uses a twostep procedure - an expectation and a maximization step-to identify model parameters that maximize the likelihood function for a given set of data. A thorough explanation of the algorithm is given by Dempster et al. (8); a more intuitive description is given by Singer et al. (30). The likelihood function of the LC model can be written as (22)

$\mathcal{L}=\prod_{j=1}^{N}\left(\eta \prod_{i=1}^{d} \pi_{i 1}^{x_{i j}}\left(1-\pi_{i 1}\right)^{1-x_{i j}}+(1-\eta) \prod_{i=1}^{d} \pi_{i 0}^{x_{i j}}\left(1-\pi_{i 0}\right)^{1-x_{i j}}\right)$

where $\eta=\operatorname{Pr}(y=1)$ is the latent class membership probability representing the unconditional probability that a sample is in the infected latent class, $\pi_{i 1}$ and $\pi_{i 0}$ are the item-response probabilities and are conditional on latent class membership, the $x_{i j}$ s are the results of the $i$ th diagnostic test on the $j$ th observation and are directly observed, $d$ is the total number of diagnostic tests, and $N$ is the total number of observations. In this study, $d=3$ where $i=$ 1,2 , or 3 indicates visual assessment of symptoms, TB, and RTPCR, respectively. The manifest variables $\left(x_{1 j}, x_{2 j}\right.$, and $\left.x_{3 j}\right)$ yield binary results such that $x_{i j}=0$ represents a negative test result and $x_{i j}=1$ represents a positive test result for test $i$ and sample $j$ for each of the $N$ samples. Each item-response probability, $\pi_{i 1}$ and $\pi_{i 0}$, is the probability of a particular outcome of the manifest variables (test results) conditional on belonging to the specified latent class for infection status (healthy or infected). From the model, $\pi_{i 1}$ is the conditional probability $\operatorname{Pr}\left(x_{i}=1 \mid y_{j}=1\right)$ and represents the sensitivity of test $i$, and $1-\pi_{i 0}$ is the conditional probability $\operatorname{Pr}\left(x_{i}=\right.$ $\left.0 \mid y_{j}=0\right)$ and is referred to as the specificity of test $i$. Finally, the
LCA model produces an estimate of disease incidence because $\eta$ is estimated by the proportion of individuals in the sample expected to be in the SqVYV-infected latent class. The LCA model makes no assumptions about the distributions of the manifest variables other than that of local independence, which specifies that, within each latent class, the manifest variables $\left(x_{i j} \mathrm{~s}\right)$ are mutually independent (23).

The latent class model can be extended to account for the effects of a categorical grouping variable $(6,7)$. A number of outcomes are possible when the latent structures of two or more groups are compared. Here, we start with what is referred to as a "heterogeneous, unrestricted $T$-class model", where it is assumed that the number of latent classes is identical among groups (two in our case, healthy and infected) but both the latent class and itemresponse probabilities have no other restrictions (27). When a grouping variable is introduced into the model, differences may occur in both the item-response probabilities (i.e., test sensitivities and specificities) and latent class membership probabilities (i.e., incidence of infection or noninfection), or only in the latent class membership probabilities (7). If the item-response probabilities do not vary statistically among the members of the grouping variable, the grouping variable is considered to be measurement invariant. When the item-response probabilities are found to be measurement invariant, the grouping variable can be removed from the model in favor of a more parsimonious model. Alternatively, the item-response probabilities can be constrained to be equivalent across the latent classes; therefore, only the latent class probabilities are estimated for each group member. When measurement invariance does not hold, one or more of the item-response probabilities varies across group members and the approach taken is to either allow all the item-response probabilities to be freely estimated or constrain various subsets of the item-response probabilities and allow the remainder to be estimated freely. Measurement invariance can also apply to the latent class probabilities. If both the latent class and item-response probabilities are found to be measurement invariant with respect to the addition of a grouping variable, the grouping variable is usually removed from the model (7).

To test for measurement invariance among the item-response probabilities, one compares the model where the item-response probabilities (i.e., the sensitivities and specificities) are restricted to be equal across groups to the model where the item-response probabilities are estimated freely. To test for measurement invariance in the latent class probabilities, one compares the model where the latent class probabilities are restricted to be equal and the item-response probabilities are restricted to be equal to the model where only the item-response probabilities are restricted to be equal. In both cases, the models are nested, allowing a likelihood-ratio test to be used to look for evidence of measurement invariance among the item-response or latent class probabilities. $G^{2}$ is the likelihood ratio statistic and is calculated by

$$
G^{2}=2 \Sigma_{x} o b s \log (o b s / \exp )
$$

where $\boldsymbol{x}$ represents a response pattern and $o b s$ and $\exp$ are the observed and the expected counts, respectively, as predicted by the model for the eight possible response patterns. The difference between the two likelihoods (i.e., $G_{\Delta}{ }^{2}=G_{1}{ }^{2}-G_{2}{ }^{2}$ ) is distributed as a $\chi^{2}$ variable with $d f=d f_{2}-d f_{1}$, where the 1 and 2 subscripts refer to two nested models, $\Delta$ refers to the difference in loglikelihoods, and $d f_{1}$ and $d f_{2}$ are the degrees of freedom for the two models under comparison. Essentially, the test is used to determine whether the addition of the grouping variable improves the overall fit of the model. When measurement invariance does not hold, the $\chi^{2}$ value is large, which is an indication that the sensitivities and specificities of the diagnostic tests vary by the mem- 
bers of the grouping variable and should be estimated separately for each member (or subset of members) of the group. Additionally, one can assess the fit of competing models, with the Akaike information criterion (AIC) $=G^{2}+2 P$ and the Bayesian information criterion (BIC) $=G^{2}+\log (N) P$, where $P$ is the number of model parameters and $N$ is the total sample size (7). The AIC does not penalize the number of parameters as strongly as the BIC. For both criteria, a smaller value represents a more optimal balance between model fit and parsimony.

In this study, four different grouping variables were examined to determine whether the item-response or latent class probabilities varied among group members. The grouping variables included tissue type (specifically, crown versus vine tissue) and distance from the crown, where distance was treated as a categorical variable by placing each of the seven vine segments into its own distance class and classes were labeled in order beginning with the portion of the vine closest to the crown (A, the first distance class) up to the vine tip ( $\mathrm{H}$, the last distance class). Also examined was the host effect or genus, which included the genera Citrullus, Cucurbita, and Cucumis (Table 1). A fourth level was created that included the genera Benincasa hispida, Trichosanthes anguina, Lageneria siceraria, and Momordica charantia. The plants species representing these genera do not produce symptoms following inoculation with SqVYV and the level was named "asymptomatic". The final grouping variable (habitat) was used to categorize plants as either field- or greenhouse-grown plants.

In practice, what is usually of most interest is the probability that a subject is truly infected given a particular test response pattern. This is known as the posterior probability or, more commonly, the positive predictive value (PPV). In the context of LCA, the PPV for being in the SqVYV-infected latent class (i.e., $y=1$ ) conditional on observing a test response pattern, $\boldsymbol{x}$, can be found using Bayes' theorem (7):

$$
\operatorname{Pr}(y=1 \mid \boldsymbol{x})=\frac{\operatorname{Pr}(y=1) \cdot \operatorname{Pr}(\boldsymbol{x} \mid y=1)}{\operatorname{Pr}(y=1) \cdot \operatorname{Pr}(\boldsymbol{x} \mid y=1)+\operatorname{Pr}(y=0) \cdot \operatorname{Pr}(\boldsymbol{x} \mid y=0)}
$$

For example, say plants test positive for SqVYV by symptoms and RT-PCR but not by TB, giving $\boldsymbol{x}=\left(x_{1}=1, x_{2}=0, x_{3}=1\right)$. The PPV is calculated:

$$
=\frac{\eta \cdot \pi_{11} \cdot\left(1-\pi_{21}\right) \cdot \pi_{31}}{\eta \cdot \pi_{11} \cdot\left(1-\pi_{21}\right) \cdot \pi_{31}+(1-\eta) \cdot \pi_{10} \cdot\left(1-\pi_{20}\right) \cdot \pi_{30}}
$$

where $\pi_{i 1}$ is the true positive proportion or the sensitivity of test $i$, $\pi_{i 0}$ is the true negative proportion of test $i$, such that $\left(1-\pi_{i 0}\right)$ is the specificity of test $i$, and $\eta$ is the incidence of disease or the probability of being in the infected latent class; all parameters are estimated from the data. For the response pattern given in the example, the term $\operatorname{Pr}(x \mid y=1)$ is the product of the probabilities of positive test results for tests $x_{1}$ and $x_{3}\left(\pi_{11}\right.$ and $\pi_{31}$, respectively) and a negative test result for test $x_{2}\left(1-\pi_{21}\right)$, conditional on being in infected latent class. The term $\operatorname{Pr}(\boldsymbol{x} \mid y=0)$ is the product of the

TABLE 2. Number of samples testing positive $(\mathrm{P})$ or negative $(\mathrm{N})$ for the eight response patterns possible with three diagnostic tests: reverse-transcription polymerase chain reaction (RT-PCR), visual assessment of symptoms, and tissue blot $(\mathrm{TB})(n=663)$

\begin{tabular}{lcccc}
\hline RT-PCR & Symptom & TB & Number of samples & Total (\%) \\
\hline P & P & P & 99 & 14.9 \\
P & P & N & 55 & 8.3 \\
P & N & P & 238 & 35.9 \\
P & N & N & 94 & 14.2 \\
N & P & P & 9 & 1.4 \\
N & P & N & 4 & 0.6 \\
N & N & P & 64 & 9.6 \\
N & N & N & 100 & 15.1 \\
\hline
\end{tabular}

probabilities of a positive test results for tests $x_{1}$ and $x_{3}\left(\pi_{10}\right.$ and $\pi_{30}$, respectively) and a negative test result for test $x_{2}\left(1-\pi_{20}\right)$, conditional on being in the noninfected latent class. The probability of being in the noninfected latent class; that is, $\operatorname{Pr}(y=$ $0)$ is $(1-\eta)$.

We used PROC LCA in SAS (version 9.1; SAS Institute, Cary, $\mathrm{NC}$ ) to fit the latent class models by treating disease status as a two-class latent variable $(23,24)$. PROC LCA is an add-on procedure for SAS that can be obtained free-of-charge through Pennsylvania State University's Methodology Center's website (http://methodology.psu.edu/ra/lca). The iterative model fitting procedure was carried out with 20 different starting values for the parameters in order to avoid converging to local maxima of the likelihood function (24). Similarly, a data-derived prior on the item-response probabilities (i.e., $\pi_{i 1}$ and $\pi_{i 0}$ ) was invoked to improve estimation by biasing them away from solutions in which some $\pi_{i 1} \mathrm{~s}$ or $\pi_{i 0} \mathrm{~s}$ are zero or one. This is important if standard errors are desired (24). The effects of the grouping variables and determination of the posterior probabilities were also determined via PROC LCA. The SAS code (along with the data for most analyses) is provided online as a supplmentary file. Any modification to the standard analysis or approach is described in Results and shown in the supplementary material. (Note: the authors of PROC LCA define the latent class membership and itemresponse probabilities using the symbols $\gamma$ and $\rho$ as opposed to $\eta$ and $\pi$ as done here.)

\section{RESULTS}

Observations. All 1,400 samples were visually assessed for disease but, of these samples, $129(9.2 \%)$ were not tested by TB and $608(43.4 \%)$ were not tested by RT-PCR. In some cases, RTPCR was done only if the other tests were contradictory. Samples that did not undergo all three tests were excluded from the analysis reported here, leaving 663 samples in total (Table 1). Of the $n=663$ samples, 472 were from 59 greenhouse-grown plants and 191 were from 24 field-grown plants. Of the total sample, $167(25.2 \%)$ tested positive by visual inspection, $410(61.8 \%)$ tested positive by TB, and $486(73.3 \%)$ tested positive by RT-PCR (Table 2). Only 199 of $663(30 \%)$ of the samples had agreement among all three tests (i.e., results were either all positive or all negative).

Baseline model. With $d=3$ diagnostic tests, each giving two possible results, there are $2^{d}=8$ response patterns (Table 2), giving $8-1=7$ degrees of freedom. The baseline model (i.e., the latent class model with no grouping variables) (Table 3, model 1.0) was fully saturated because there were seven parameters to estimate (three sensitivities, three specificities, and an estimate of disease incidence). For this model, PROC LCA was able to produce the point estimates but was not able to calculate the standard errors using the EM algorithm. To generate standard errors, we constrained the estimates of specificity for RT-PCR and visual assessment to be equal (Table 3, model 1.1), based on nearequivalent point estimates of specificity generated from the unconstrained baseline model ( 0.95 and 0.99 for visual assessment and RT-PCR, respectively). The constrained baseline model produced an estimate of incidence of $\widehat{\eta}=0.78$ with a standard error of 0.03, which is the probability of being in the SqVYV-infected latent class (Table 4). As a comparison for this model only, the unconstrained baseline model was fit to the data using the specialty package Tests in the Absence of a Gold Standard) as part of the R statistical software, because this routine can generate percentile bootstrap confidence intervals, which do not rely on standard errors for calculation (28). The point estimates of the sensitivities and specificities for the unconstrained model were within 0.02 of the estimates in the constrained model, and the bootstrap confidence intervals (Table 5) were identical or slightly narrower than the normal-based $95 \%$ confidence intervals (Table 
4). In the baseline model, visual assessment of symptoms had the overall lowest sensitivity for detection of SqVYV infection, whereas RT-PCR had the highest (Tables 4 and 5). The specificities of visual assessment and RT-PCR were constrained to be equal and were estimated at 0.98 (Table 4). The estimated specificity of TB was 0.65 .

Grouping variables. For the grouping variables, a likelihood ratio test showed that measurement invariance of the itemresponse probabilities did not hold for tissue type $\left(G_{\Delta}{ }^{2}=22.1\right.$, $d f=6, \operatorname{Pr}=0.001)$ (Table 3, model 2.1), meaning that significant differences in test performance were found between crown and vine tissue. The specificity of TB with crown tissue was considerably lower than that of vine tissue $(0.27$ versus 0.70$)$ (Table 6$)$. Similarly, the specificity of RT-PCR with crown tissue was also lower than that for vine tissue ( 0.46 versus 0.94 ) (Table 6$)$. In this model, the estimate of incidence of SqVYV in vine tissue was greater than the estimate in crown tissue $(\widehat{\eta}=0.77$ versus $\widehat{\eta}=$ 0.56 , respectively) based on a likelihood ratio test of measurement invariance of the latent class probabilities $\left(G_{\Delta}^{2}=26.2, d f=\right.$ 1, $\operatorname{Pr}<0.0001$ ) (Table 3, model 2.2). A likelihood-ratio test showed that measurement invariance for the item-response probabilities held for distance from the crown $\left(G_{\Delta}{ }^{2}=41.9, d f=36\right.$, $\operatorname{Pr}=0.23)\left(\right.$ Table 3, model 3.1) and habitat $\left(G_{\Delta}^{2}=7.25, d f=6\right.$, $\operatorname{Pr}=0.30)$ (Table 3, model 4.1), meaning that test sensitivities and specificities did not vary along the length of the vine or between plants grown in the field or greenhouse for any of the three tests. However, there was a significant difference in the latent class probabilities (i.e., disease incidence) between field- and greenhouse-grown plants ( 0.88 versus 0.66 , respectively) (Table 3 , model 4.2) but not for distance from the crown (Table 3 , model 3.2). The former is likely a reflection of differences in the environmental conditions between field and greenhouse grown plants.

A likelihood ratio test showed that measurement invariance of the item-response probabilities did not hold for genera $\left(G_{\Delta}{ }^{2}=\right.$ 153.2, $d f=18, \operatorname{Pr}<0.0001$ ) (Table 3, model 5.1), meaning that test performance varied among plant genera (Table 7). Similarly, a likelihood ratio test showed that measurement invariance did not hold for the latent class probabilities $\left(G_{\Delta}^{2}=91.4, d f=3, \operatorname{Pr}<\right.$ 0.0001) (Table 3, model 5.2), meaning that disease incidence varied among plant genera as well (Table 7). In search for the most parsimonious model containing plant genera, multiple sets of constraints were investigated: (i) equality among symptom specificity (Table 3, model 5.3), (ii) equality among RT-PCR sensitivity (Table 3, model 5.4), and (iii) the combination of (i) and (ii) (Table 3, model 5.5). Based on a combination of model results, their fit statistics, and the use of subjective judgment, the model with constrained RT-PCR sensitivity (model 5.4) was selected and the parameter estimates are shown in Table 7. The estimate of RT-PCR sensitivity across genera was 0.99 , while the specificities were generally low among the genera except for Citrullus, which had a specificity of 0.96 (Table 7). The specificity of symptoms was greater than 0.85 among genera, with Citrullus and cultivars within the asymptomatic grouping achieving perfect specificity. The sensitivity for symptoms was very poor for the genus Cucumis and the asymptomatic grouping; the latter was clearly expected, given that infected plants within this group show no symptoms. TB sensitivity was generally better than its specificity among genera (Table 7).

Posterior probabilities. Tables 8 and 9 show the PPVs for the tissue type and genus groupings, respectively. For crown tissue, visibly diseased crowns that tested positive by RT-PCR had the greatest PPVs (Table 8). A positive TB result did not significantly impact the probabilities. Indeed, a sole positive by TB (with the

TABLE 4. Estimates of the sensitivities, specificities, their associated standard errors, and the lower and upper bounds of a 95\% confidence interval (CI) determined with a latent class analysis (LCA) model for three diagnostic tests of Squash vein yellowing virus (SqVYV) infection ${ }^{\mathrm{a}}$

\begin{tabular}{lcccc}
\hline Test $^{\mathrm{b}}$ & Sensitivity & $95 \% \mathrm{CI}$ & Specificity & $95 \% \mathrm{CI}$ \\
\hline RT-PCR & 0.937 & $0.88,0.99$ & 0.979 & $0.93,1.00$ \\
& $(0.027)$ & & $(0.024)$ & \\
Symptom & 0.319 & $0.28,0.36$ & 0.979 & $0.93,1.00$ \\
& $(0.021)$ & & $(0.024)$ & \\
TB & 0.698 & $0.66,0.74$ & 0.654 & $0.55,0.76$ \\
& $(0.021)$ & & $(0.053)$ & \\
\hline
\end{tabular}

a Estimated parameters were derived from the baseline latent class model $(n=$ 663 ) with the add-on SAS procedure PROC LCA; the probability of being in the SqVYV-infected latent class (= disease incidence) was estimated to be $\widehat{\eta}=0.777(0.03)$ and for being in the non-SqVYV-infected latent class (1 $\widehat{\eta})=0.223(0.03)$. Values in parentheses are the standard errors.

b Reverse-transcription polymerase chain reaction (RT-PCR), visual assessment of symptoms (Symptom), and tissue blot (TB).

TABLE 3. Goodness-of-fit and the likelihood ratio statistics for latent class models fit in this study

\begin{tabular}{|c|c|c|c|c|c|c|c|c|}
\hline \multirow[b]{2}{*}{ Model (constraints) ${ }^{\mathrm{a}}$} & \multirow[b]{2}{*}{$d f$} & \multicolumn{3}{|c|}{ Goodness of fit statistics ${ }^{b}$} & \multicolumn{4}{|c|}{ Likelihood ratio test ${ }^{\mathrm{c}}$} \\
\hline & & $G^{2}$ & $\mathrm{AIC}$ & BIC & Comp. & $\Delta G^{2}$ & $\Delta d f$ & $P$ value \\
\hline 1.0 Baseline & 0 & 3.44 & 17.44 & 48.92 & $\ldots$ & $\ldots$ & $\ldots$ & $\ldots$ \\
\hline 1.1 Baseline/constrained ${ }^{\mathrm{d}}$ & 1 & 3.26 & 15.26 & 42.24 & $\ldots$ & $\ldots$ & $\ldots$ & $\ldots$ \\
\hline 2.0 Tissue type & 1 & 3.65 & 31.65 & 94.60 & $\ldots$ & $\ldots$ & $\ldots$ & $\ldots$ \\
\hline 2.1 Tissue type (IR) & 7 & 25.70 & 41.70 & 77.68 & 2.0 & 22.05 & 6 & 0.001 \\
\hline 2.2 Tissue type (IR+LC) & 8 & 51.88 & 65.88 & 97.36 & 2.1 & 26.18 & 1 & $<0.0001$ \\
\hline 3.0 Distance & 6 & 15.44 & 113.44 & 327.31 & $\ldots$ & $\ldots$ & $\ldots$ & $\ldots$ \\
\hline 3.1 Distance (IR) & 42 & 57.31 & 83.31 & 140.06 & 3.0 & 41.87 & 36 & 0.23 \\
\hline 3.2 Distance (IR+LC) & 48 & 64.22 & 78.22 & 108.77 & 3.1 & 6.91 & 6 & 0.33 \\
\hline 4.0 Habitat & 1 & 8.72 & 36.72 & 99.68 & $\ldots$ & $\ldots$ & $\ldots$ & $\ldots$ \\
\hline 4.1 Habitat (IR) & 7 & 15.97 & 31.97 & 67.94 & 4.0 & 7.25 & 6 & 0.30 \\
\hline 4.2 Habitat (IR+LC) & 8 & 45.39 & 59.39 & 90.87 & 4.1 & 29.42 & 1 & $<0.0001$ \\
\hline 5.0 Genus & 3 & 3.44 & 59.44 & 185.35 & $\ldots$ & $\ldots$ & $\ldots$ & $\ldots$ \\
\hline 5.1 Genus (IR) & 21 & 156.62 & 176.62 & 221.59 & 5.0 & 153.18 & 18 & $<0.0001$ \\
\hline 5.2 Genus (IR+LC) & 24 & 249.04 & 263.04 & 294.52 & 5.1 & 91.42 & 3 & $<0.0001$ \\
\hline 5.3 Genus (Sym SP) & 6 & 5.79 & 55.79 & 168.21 & 5.0 & 2.35 & 3 & 0.50 \\
\hline 5.4 Genus (PCR SE) & 6 & 4.32 & 54.32 & 166.74 & 5.0 & 0.88 & 3 & 0.83 \\
\hline 5.5 Genus (Sym SP and PCR SE) & 9 & 12.60 & 56.60 & 155.53 & 5.0 & 9.16 & 6 & 0.16 \\
\hline
\end{tabular}

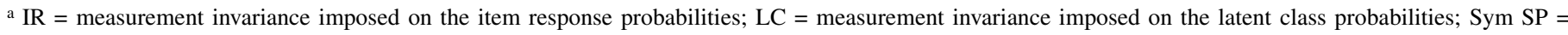
symptom specificity constrained to be equal across genus levels; PCR SE = reverse-transcription polymerase chain reaction (RT-PCR) sensitivity constrained to be equal across genus levels.

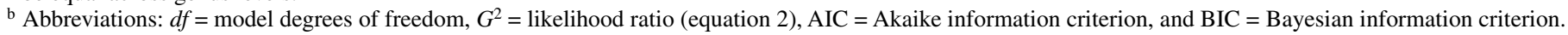

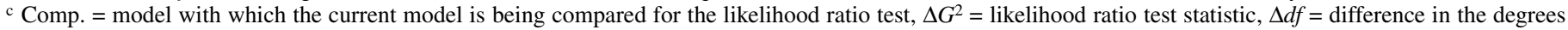
of freedom between the two models being compared, and $P$ value for the likelihood ratio statistic.

d Baseline model constrained to hold symptoms and RT-PCR specificities to be equal. 
other two tests giving negative results) was just as likely to indicate a noninfected crown as when all three tests were negative. For vine tissue, the combination of positive test results from any two tests (with the remaining test being negative) resulted in PPVs $>0.952$ (Table 8). A sole positive by RT-PCR, with the other two tests giving negative results, gave a PPV of 0.94 . Results also showed that symptomatic plants with negative TB and RT-PCR

TABLE 5. Estimates of the sensitivities, specificities, and the lower and upper bounds of a 95\% percentile bootstrap confidence interval (CI) determined with a latent class analysis (LCA) model for three diagnostic tests of Squash vein yellowing virus (SqVYV) infection ${ }^{\mathrm{a}}$

\begin{tabular}{lcccc}
\hline Test $^{\mathrm{b}}$ & Sensitivity & Bootstrap CI & Specificity & Bootstrap CI \\
\hline RT-PCR & 0.922 & $0.88,0.99$ & 1.000 & $1.00,1.00$ \\
Symptom & 0.317 & $0.28,0.36$ & 0.999 & $0.93,1.00$ \\
TB & 0.693 & $0.65,0.74$ & 0.672 & $0.56,0.77$ \\
\hline
\end{tabular}

${ }^{a}$ Estimated parameters were derived from the baseline latent class model $(n=$ 663 ) using the TAGS software (28); The probability of being in the SqVYVinfected latent class ( $=$ disease incidence) and the bootstrap confidence interval were $\hat{\eta}=0.795(0.732,0.833)$.

${ }^{\mathrm{b}}$ Reverse-transcription polymerase chain reaction (RT-PCR), visual assessment of symptoms (Symptom), and tissue blot (TB). had a higher PPV than plants testing positive solely by TB $(0.79$ versus 0.27 ). This result should be interpreted with caution, however, given that there were only $n=4$ in the group where symptoms were the sole positive test, versus $n=52$ plants where TB was the sole positive.

When compared across genera, the highest PPVs were associated with three positive test results (Table 9). High PPVs in Citrullus spp. were associated with at least two positive test results, as long as one of the positives was from RT-PCR. In Cucurbita spp., high PPVs were associated with symptomatic plants testing positive by RT-PCR. The addition of a positive by TB slightly reduced the PPV. For Cucumis spp. and plants collectively labeled asymptomatic, the highest PPVs were associated with plants testing positive by both RT-PCR and TB. In these categories, there were no plants that tested positive only by TB.

\section{DISCUSSION}

LCA was used to estimate the sensitivities and specificities of visual assessment of symptoms, TB, and RT-PCR for diagnosis of SqVYV infection. Overall, results showed that RT-PCR had the highest sensitivity and specificity of the three tests. TB has been

TABLE 6. Estimates of sensitivities, specificities, and their associated standard errors for three diagnostic tests of Squash vein yellowing virus (SqVYV) infection determined from a latent class model where measurement invariance was not imposed across the tissue-type grouping variable

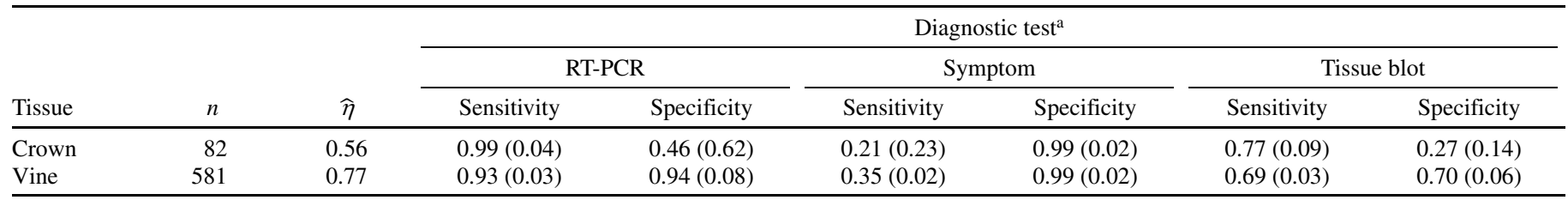

${ }^{a}$ RT-PCR $=$ reverse-transcription polymerase chain reaction, $n=$ number of samples, $\widehat{\eta}$ = probability of being in the SqVYV-infected latent class $(=$ disease incidence), and values in parentheses are standard errors. Sensitivity of test $i$ is the conditional probability $\operatorname{Pr}\left(x_{i}=1 \mid y=1\right)$ and is denoted in the text as the itemresponse probability $\pi_{i 1}$; the specificity of test $i$ is the conditional probability $\operatorname{Pr}\left(x_{i}=0 \mid y=0\right)$ and is denoted in the text as the item-response probability $\left(1-\pi_{i 0}\right)$.

TABLE 7. Estimates of sensitivities, specificities, and their associated standard errors for three diagnostic tests of Squash vein yellowing virus (SqVYV) infection estimated from a latent class model where measurement invariance was not imposed across the genus grouping variable

\begin{tabular}{|c|c|c|c|c|c|c|c|c|}
\hline \multirow[b]{3}{*}{ Genus } & \multirow[b]{3}{*}{$n$} & \multirow[b]{3}{*}{$\widehat{\eta}$} & \multicolumn{6}{|c|}{ Diagnostic test $^{\mathrm{a}}$} \\
\hline & & & \multicolumn{2}{|c|}{ RT-PCR } & \multicolumn{2}{|c|}{ Symptom } & \multicolumn{2}{|c|}{ Tissue blot } \\
\hline & & & Sensitivity & Specificity & Sensitivity & Specificity & Sensitivity & Specificity \\
\hline Citrullus & 168 & 0.62 & $0.99(0.02)$ & $0.96(0.05)$ & $0.36(0.05)$ & $0.99(0.01)$ & $0.88(0.04)$ & $0.75(0.06)$ \\
\hline Cucurbita & 247 & 0.55 & $0.99(0.02)$ & $0.53(0.27)$ & $0.73(0.25)$ & $0.85(0.05)$ & $0.55(0.05)$ & $0.40(0.06)$ \\
\hline Cucumis & 144 & 0.67 & $0.99(0.02)$ & $0.53(0.34)$ & $0.11(0.04)$ & $0.91(0.06)$ & $0.92(0.14)$ & $0.50(0.10)$ \\
\hline Asymptomatic & 80 & 0.44 & $0.99(0.02)$ & $0.43(0.31)$ & $0.00(0.01)$ & $0.99(0.01)$ & $0.73(0.43)$ & $0.73(0.09)$ \\
\hline
\end{tabular}

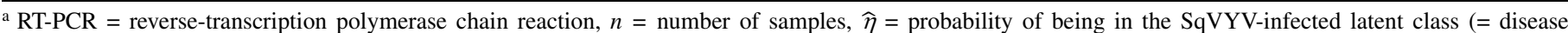
incidence), and values in parentheses are standard errors. The sensitivity of test $i$ is the conditional probability $\operatorname{Pr}\left(x_{i}=1 \mid y=1\right)$ and is denoted in the text as the item-response probability $\pi_{i 1}$; the specificity of test $i$ is the conditional probability $\operatorname{Pr}\left(x_{i}=0 \mid y=0\right)$ and is denoted in the text as the item-response probability $\left(1-\pi_{i 0}\right)$. Sensitivity of RT-PCR was constrained to be equal across genus levels.

TABLE 8. Positive predictive value (PPV) (i.e., posterior probability) of a sample being classified as infected with Squash vein yellowing virus (SqVYV) based on a diagnostic test response pattern from tests on crown or vine tissue

\begin{tabular}{|c|c|c|c|c|c|c|}
\hline & & & \multicolumn{4}{|c|}{ Tissue type } \\
\hline \multicolumn{3}{|c|}{ Test response $(\boldsymbol{x})^{\mathrm{a}}$} & \multicolumn{2}{|c|}{ Crown } & \multicolumn{2}{|c|}{ Vine } \\
\hline RT-PCR & Symptom & TB & $n$ & $\operatorname{Pr}(y=1 \mid x)^{\mathrm{b}}$ & $n$ & $\operatorname{Pr}(y=1 \mid x)$ \\
\hline $\mathrm{P}$ & $\mathrm{P}$ & $\mathrm{N}$ & 3 & 0.977 & 52 & 0.999 \\
\hline $\mathrm{P}$ & $\mathrm{N}$ & $\mathrm{P}$ & 43 & 0.663 & 195 & 0.987 \\
\hline $\mathrm{P}$ & $\mathrm{N}$ & $\mathrm{N}$ & 12 & 0.613 & 82 & 0.938 \\
\hline $\mathrm{N}$ & $\mathrm{P}$ & $\mathrm{P}$ & $-^{c}$ & 0.380 & 9 & 0.952 \\
\hline $\mathrm{N}$ & $\mathrm{N}$ & $\mathrm{N}$ & 5 & 0.018 & 95 & 0.068 \\
\hline
\end{tabular}

${ }^{a}$ Reverse-transcription polymerase chain reaction (RT-PCR), visual assessment of symptoms (Symptom), and tissue blot $(\mathrm{TB}) . \mathrm{P}=$ positive test result, $\mathrm{N}=$ negative test result, $n=$ number of samples with that particular response patterns.

${ }^{\mathrm{b}} \operatorname{Pr}(y=1 \mid x)$ is the posterior probability of belonging to the SqVYV-infected latent class given test response $x$. The value of $\widehat{\eta}$ used to calculate PPV was 0.56 for crown tissue and 0.77 for vine tissue.

${ }^{c}$ No observations for this combination of test results existed in the data set; however, it is still possible to calculate a PPV. 
used as the standard high-throughput test to diagnose SqVYV infection in watermelon, even though little was known of its performance characteristics on other cucurbit species. Although TB had better sensitivity than visual inspection of symptoms for detection of SqVYV infection, the visual assessment of symptoms was more specific than TB and, thus, a better indicator of noninfection. The fact that symptoms had such poor sensitivity is a reflection of the lag time between infection and symptom development, the fact that some cucurbits are slow to develop symptoms of SqVYV infection, and the fact that many non-watermelon species of cucurbits exhibit either no or very mild symptoms; all commercial watermelon cultivars exhibit distinct symptoms of SqVYV infection. In contrast, the high specificity of symptoms relative to TB is an indicator that symptoms of SqVYV infection are easily identifiable (at least by a trained rater with this set of plants) whereas the interpretation of TB test results can be problematic. For example, the species $L$. siceraria consistently generates positive results by $\mathrm{TB}$ regardless of SqVYV infection status $(26,36)$.

Test performance varied with respect to the type of plant tissue being tested. RT-PCR and TB had better sensitivity but poorer specificity for diagnosing SqVYV infection in crown tissue than it did in vine tissue, whereas symptoms had very poor sensitivity but excellent specificity in both tissues. The higher sensitivity of RT-PCR and TB in crown tissue supports earlier recommendations that crown tissue should be the preferred tissue for testing $(2,32)$. The reasons for the increased sensitivity of RT-PCR and TB in crown tissue may be due to a higher titer of virus or viral RNA in the crown tissue of infected plants than in vine tissue, which may be related to the host-pathogen interaction. It is also possible that the viral nucleic acids extracted from declining vine tissue is of poor quality and adversely affects test performance. In contrast, RT-PCR and, particularly, TB are less specific than visual assessment of symptoms in crown tissue, leading to a higher false-positive rate. This is easily explained for $\mathrm{TB}$, where the subjective interpretation of the blots is difficult on overexposed blots. The poor specificity of RT-PCR, however, is more difficult to explain and likely an artifact of the analysis; this will be discussed in greater detail below.

Test performance also varied by genus. When forming the genus grouping, we were aware that it might not be reasonable to assume that all species within a genus respond identically to each of the tests. However, it was decided to create a genus rather than a species grouping because many of the species had only a small number of samples to test because of the smaller number of plants suitable for inoculation (Table 1), and the knowledge that small sample sizes result in low statistical power. Although sample size played a role, $B$. hispida, $T$. anguina, $L$. siceraria, and $M$. charantia were combined into a single asymptomatic group because plants of these species do not produce symptoms following inoculation with SqVYV. Results showed that for the cultivars analyzed in this study, the standard errors of the sensitivities and specificities (i.e., the item-response probabilities) were relatively small for most levels within the genus grouping, suggesting that each of the three tests performed similarly among species within a genus (Table 7). A notable exception, however, were the specificities of RT-PCR for the genera Cucurbita and Cucumis and the asymptomatic grouping; this is related to the generally poor specificity for RT-PCR in crown tissue noted above and likely affected the point estimates of the specificity (i.e., they are lower than expected, given the values in Table 4).

LCA is well established in statistics and has been applied many times in disciplines outside of plant pathology. Latent class (structure) analysis was developed by the preeminent sociologist Paul Lazarsfeld (25), and Hui and Walter (20) most notably applied the latent class methodology to the diagnostic test setting. The LCA approach is commonly used in both medical and veterinary epidemiology for evaluation of diagnostic test performance. LCA

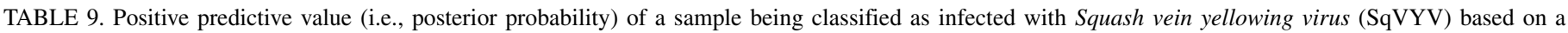
diagnostic test response pattern from tests on different genera of cucurbits

\begin{tabular}{|c|c|c|c|c|c|c|c|c|c|c|}
\hline & & & \multicolumn{6}{|c|}{ Genus } & & \\
\hline \multicolumn{3}{|c|}{ Test response $(\boldsymbol{x})^{\mathrm{a}}$} & \multicolumn{2}{|c|}{ Citrullus } & \multicolumn{2}{|c|}{ Cucurbita } & \multicolumn{2}{|c|}{ Cucumis } & \multicolumn{2}{|c|}{ Asymptomatic } \\
\hline RT-PCR & Symptom & TB & $n$ & $\operatorname{Pr}(y=1 \mid \boldsymbol{x})^{\mathrm{b}}$ & $n$ & $\operatorname{Pr}(y=1 \mid \boldsymbol{x})$ & $n$ & $\operatorname{Pr}(y=1 \mid x)$ & $n$ & $\operatorname{Pr}(y=1 \mid x)$ \\
\hline $\mathrm{P}$ & $\mathrm{P}$ & $\mathrm{P}$ & 31 & 0.999 & 57 & 0.920 & 11 & 0.906 & $\ldots$ & $\ldots$ \\
\hline $\mathrm{P}$ & $\mathrm{P}$ & $\mathrm{N}$ & 6 & 0.996 & 48 & 0.934 & 1 & 0.455 & $\ldots$ & $\ldots$ \\
\hline $\mathrm{P}$ & $\mathrm{N}$ & $\mathrm{P}$ & 60 & 0.989 & 48 & 0.428 & 88 & 0.885 & 42 & 0.788 \\
\hline $\mathrm{P}$ & $\mathrm{N}$ & $\mathrm{N}$ & 8 & 0.807 & 33 & 0.479 & 17 & 0.401 & 36 & 0.338 \\
\hline $\mathrm{N}$ & $\mathrm{P}$ & $\mathrm{P}$ & $-^{c}$ & 0.683 & 7 & 0.093 & 2 & 0.079 & $\ldots$ & $\ldots$ \\
\hline $\mathrm{N}$ & $\mathrm{P}$ & $\mathrm{N}$ & $-^{c}$ & 0.089 & 3 & 0.112 & 1 & 0.007 & $\ldots$ & $\ldots$ \\
\hline $\mathrm{N}$ & $\mathrm{N}$ & $\mathrm{P}$ & 16 & 0.037 & 29 & 0.007 & 12 & 0.065 & 7 & 0.048 \\
\hline $\mathrm{N}$ & $\mathrm{N}$ & $\mathrm{N}$ & 47 & 0.002 & 22 & 0.008 & 12 & 0.006 & 19 & 0.007 \\
\hline
\end{tabular}

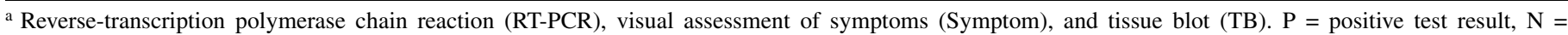
negative test result, $n=$ number of samples with that particular response patterns.

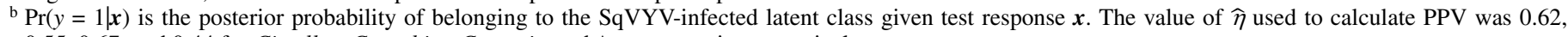
0.55, 0.67, and 0.44 for Citrullus, Cucurbita, Cucumis, and Asymptomatic, respectively.

${ }^{\mathrm{c}}$ No observations for this combination of test results existed in the data set; however, it is still possible to calculate a PPV.

TABLE 10. Sensitivity and specificity for test results combined in parallel or in serial

\begin{tabular}{|c|c|c|c|c|c|c|c|c|}
\hline \multirow[b]{3}{*}{ Test, tissue ${ }^{a}$} & \multicolumn{8}{|c|}{ Test grouping ${ }^{\mathrm{b}}$} \\
\hline & \multicolumn{2}{|c|}{ PCR-Sym } & \multicolumn{2}{|c|}{ PCR-TB } & \multicolumn{2}{|c|}{ Sym-TB } & \multicolumn{2}{|c|}{ PCR-Sym-TB } \\
\hline & Sensitivity & Specificity & Sensitivity & Specificity & Sensitivity & Specificity & Sensitivity & Specificity \\
\hline \multicolumn{9}{|l|}{ Parallel } \\
\hline Crown & 0.992 & 0.455 & 0.998 & 0.124 & 0.818 & 0.267 & 0.998 & 0.123 \\
\hline Vine & 0.955 & 0.931 & 0.978 & 0.658 & 0.799 & 0.693 & 0.986 & 0.651 \\
\hline \multicolumn{9}{|l|}{ Serial } \\
\hline Crown & 0.208 & 0.995 & 0.762 & 0.606 & 0.162 & 0.993 & 0.160 & 0.996 \\
\hline Vine & 0.326 & 0.999 & 0.642 & 0.982 & 0.242 & 0.997 & 0.225 & 0.999 \\
\hline
\end{tabular}

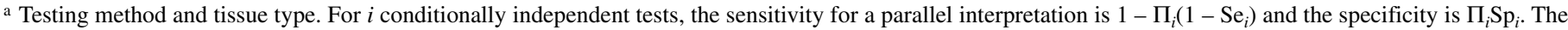
sensitivity for a serial interpretation is $\Pi_{i} \mathrm{Se}_{i}$ and the specificity is $1-\Pi_{i}\left(1-\mathrm{Sp}_{i}\right)$.

b Pairing of tests: $\mathrm{PCR}=$ reverse-transcription polymerase chain reaction, $\mathrm{TB}=$ tissue blot, and Sym $=$ visual assessment of symptoms. 
has been used recently in plant pathology to estimate test parameters for PCR-based tests of citrus greening in the United States (31) and for double-antibody sandwich enzyme-linked immunosorbent assay (ELISA) and RT-PCR tests for Plum pox virus in Spain (33). In the diagnostic setting, the use of LCA is done under the assumption that the diagnostic tests are imperfect measures of an underlying latent structure (that is, the unobserved infection status of the subjects) and that the observed response patterns of the diagnostic tests can be explained by the latent structure $(29,37)$. In the diagnostic setting, we presume to know the underlying latent structure and, thus, define a two-class latent variable (infected and noninfected classes) to explain the test results. There are other statistical options available to estimate test properties in the absence of a gold standard test but most require at least some information about the tests in question (10). For example, one could use an imperfect reference test to estimate diagnostic test parameters of the unknown tests if the sensitivity and specificity of the reference test are known (14). For our situation, none of the test parameters for any of the diagnostic tests were known a priori.

When conducting LCA, individuals are assigned to a single class based on model output but it is the responsibility of the analyst to identify or label the classes. In this study, it seemed reasonable to assume that the class with the highest estimated incidence be labeled the SqVYV-infected class because every plant in the study was inoculated or presumed to be naturally infected. Indeed, our initial criterion for assigning latent class membership was based on observed incidence but we found that it was not always practical to assign the latent class with the highest incidence as the SqVYV-infected latent class based on the corresponding item-response probabilities. Also, when a grouping variable is involved, the item-response probabilities can vary across the levels of a grouping variable (i.e., measurement invariance does not hold) and the latent classes may have a different interpretation among group levels. This is generally where "expert knowledge" and subjective reasoning are employed, and this is illustrated with the asymptomatic grouping in Table 7 . For the asymptomatic group, if one assumed that the latent class with the highest incidence was the SqVYV-infected class, this would have resulted in an estimated incidence of $\widehat{\eta}=1-0.44=0.56$ and sensitivities of $0.57,0.002$, and 0.27 and specificities of 0.01 , 0.99 , and 0.26 for RT-PCR, symptoms, and TB, respectively. The particularly poor sensitivities and specificities for RT-PCR and TB led us to select the other latent class as the SqVYV-infected class, which gave the estimates shown in Table 7. Based on what we know about virus movement, we reasoned that the virus did not migrate through the plant from the point of inoculation and, because the diagnostic tests were applied to individual plant parts and not to the plant as a whole, that many plant parts could test negative despite the plant being inoculated.

Although the LCA model makes few assumptions, the local conditional independence assumption is an important one to verify. The local independence assumption states that, conditional on latent class membership, the observed (manifest) variables are independent. In a diagnostic setting, consider a test with a sensitivity of 0.9 used to test a population of 100 infected plants. For this test, one would expect 10 false-negative results. If we were to use a second test with a sensitivity of 0.7 to test the 10 plants that yielded false-negative results, we would expect 7 of those 10 plants to test positive if the two tests were conditionally independent. Georgiadis et al. (15) developed a Bayesian conditional dependence model for situations where the conditional independence assumption does not hold; however, the model is more difficult to fit and requires reasonably precise prior information on the values of at least some of the diagnostic-test parameters. Formann et al. (12) suggests increasing the number of latent classes to account for correlation, although it is not clear how to derive the sensitivities and specificities for the diagnostic tests when more than two classes exist. Another approach is to model the latent classes using a log-linear model, as described by Espeland and Handelman (11). A simpler approach, however, is to assume conditional independence based on the argument that the diagnostic tests measure different biological properties (5). For this study, one could convincingly argue that diagnoses based on visual assessment were largely independent from diagnoses based on RT-PCR or TB. On the other hand, RT-PCR and TB can be assumed to be at least partially dependent because they both target the SqVYV CP gene. However, according to Branscum et al. (5). if the tests have markedly different detection limits, which they do, then this dependence is not likely to be an issue. Moreover, if any test is perfectly sensitive (or specific) (Table 5, RTPCR), then the tests sensitivities (or specificities) are conditionally independent by definition (13). These properties, plus the absence of any good prior information on either TB or RT-PCR test properties, provided sufficient justification for assuming conditional independence.

The sensitivities and specificities shown in Tables 6 and 7 were used to calculate posterior probabilities of infection, and these are shown relative to tissue type and genus groupings in Tables 8 and 9 , respectively. These values are the probability of SqVYV infection for a particular set of test results. When multiple tests are available, their combined results can be used in what is referred to as either parallel or serial interpretation (13). In a parallel interpretation, a sample is considered positive if at least one test is positive. Parallel interpretation is referred to as an "or" scheme and is used to maximize sensitivity. For $i$ conditionally independent tests, the sensitivity for a parallel interpretation is $1-$ $\Pi_{i}\left(1-\pi_{i 1}\right)$ and the specificity is $\Pi_{i}\left(1-\pi_{i 0}\right)$. In a serial interpretation, a sample is considered positive if all tests are positive; otherwise, it is negative. Serial interpretation is referred to as an "and" scheme. It is used to maximize the specificity, often when the cost of a false positive is high. The sensitivity for a serial interpretation is $\Pi_{i} \pi_{i 1}$ and the specificity is $1-\Pi_{i} \pi_{i 0}$. The sensitivities and specificities for both a parallel and serial interpretation are given for all possible combinations of the diagnostic test outcomes on both crown and vine tissue (Table 10). Note that, for a parallel interpretation, the sensitivity of the combined test result is always higher than the individual tests sensitivities (Table 6). Similarly, for a serial interpretation, the specificity of the combined test result is always higher than the individual specificities of the component tests. The sensitivities and specificities shown in Table 10 can be used to calculate the posterior probabilities of infection as well. The PPV for a serial interpretation can also be obtained directly from Table 8 by considering the results where all three tests are positive and is 0.981 and 0.999 for crown and vine tissue, respectively. For a parallel interpretation, only negative test results on all three tests would be considered negative; therefore, the PPV would be obtained as $1-\operatorname{Pr}(\boldsymbol{x} \mid y=1)$ where $\boldsymbol{x}=\left(x_{1}=x_{2}=x_{3}=0\right)$. In this case, $1-0.018=0.982$ and $1-0.068=0.932$ for crown and vine tissue, respectively.

The results given here provide quantitative measurements of test performance for a range of conditions and cucurbit types, and provide the information needed to interpret test results when tests are used singly or in combination for a diagnosis. Several practical implications for SqVYV testing are apparent. First, when averaged over all cucurbits and tissues tested, RT-PCR is the superior test for confirmation of SqVYV infection. Combining RTPCR with either TB or symptoms in either parallel or serial will bolster the sensitivity or specificity, respectively. Second, when testing crown tissue, a positive by RT-PCR plus a positive by either TB or symptoms will identify infection with $\geq 98.9 \%$ probability. For vine tissue, the combination of positive test results from any two tests will identify infection with $\geq 94.6 \%$ probability. Third, across the range of cucurbits tested, the sensitivity and specificity of TB was variable. Results here suggest that TB 
remains useful for high-throughput screening of watermelon, especially when all sampling and sample preparation must be completed in the field. This could be especially important in situations where sample storage is not possible or sample movement is restricted. However, TB is prone to generating falsepositive results, particularly on cucurbits other than watermelon. Thus, the decision to use TB should be dependent on the tissue or genera of cucurbit to be tested. Future research should focus on developing field-based scouting protocols based on the performance characteristics determined here and an antibody-based test (e.g., ELISA) as a high-throughput alternative that may avoid the false-positive issues observed with $\mathrm{TB}$ in some non-Citrullus cucurbits. From an analytical perspective, it would be useful to be able to directly incorporate more than one grouping variable in the LCA model because there are often several variables that need to be considered in most diagnostic situations.

\section{LITERATURE CITED}

1. Adkins, S., McCollum, T. G., Albano, J. P., Kousik, C. S., Baker, C. A., Webster, C. G., Roberts, P. D., Webb, S. E., and Turechek, W. W. 2013. Physiological effects of Squash vein yellowing virus infection on watermelon. Plant Dis. 97:1137-1148.

2. Adkins, S., Webb, S. E., Achor, D., Roberts, P. D., and Baker, C. A. 2007. Identification and characterization of a novel whitefly-transmitted member of the family Potyviridae isolated from cucurbits in Florida. Phytopathology 97:145-154.

3. Adkins, S., Webb, S. E., Baker, C. A., and Kousik, C. S. 2008. Squash vein yellowing virus detection using nested polymerase chain reaction demonstrates that the cucurbit weed Momordica charantia is a reservoir host. Plant Dis. 92:1119-1123.

4. Adkins, S., Webster, C. G., Baker, C. A., Weaver, R., Rosskopf, E. N., and Turechek, W. W. 2009. Detection of three whitefly-transmitted viruses infecting the cucurbit weed, Cucumis melo var. dudaim, in Florida. Online. Plant Health Progress. Online publication. doi:10.1094/PHP2009-1118-01-BR

5. Branscum, A. J., Gardner, I. A., and Johnson, W. O. 2005. Estimation of diagnostic-test sensitivity and specificity through Bayesian modeling. Prev. Vet. Med. 68:145-163.

6. Clogg, C. C., and Goodman, L. A. 1984. Latent structure analysis of a set of multidimensional contingency tables. J. Am. Stat. Assoc. 79:762-771.

7. Collins, L. M., and Lanza, S. T. 2010. Latent Class and Latent Transition Analysis: With Applications in the Social, Behavioral, and Health Sciences. John Wiley \& Sons, Inc., Hoboken, NJ.

8. Dempster, A., Laird, N., and Rubin, D. 1977. Maximum likelihood from incomplete data via the EM algorithm. J. R. Stat. Soc. Ser. B. 39:1-38.

9. Egel, D. S., and Adkins, S. 2007. Squash vein yellowing virus identified in watermelon (Citrullus lanatus) in Indiana. Plant Dis. 91:1056.

10. Enøe, C., Georgiadis, M. P., and Johnson, W. O. 2000. Estimation of sensitivity and specificity of diagnostic tests and disease prevalence when the true disease state is unknown. Prev. Vet. Med. 45:61-81.

11. Espeland, M. A., and Handelman, S. A. 1989. Using latent class models to characterize and assess relative error in discrete measurements. Biometrics 45:587-599.

12. Formann, A. K. 1994. Measurement errors in caries diagnosis: Some further latent class models. Biometrics 50:865-871.

13. Gardner, I. A., Stryhn, H., Lind, P., and Collins, M. T. 2000. Conditional dependence between tests affects the diagnosis and surveillance of animal diseases. Prev. Vet. Med. 45:107-122.

14. Gart, J. J., and Buck, A. A. 1966. Comparison of a screening test and a reference test in epidemiological studies. II. A probabilistic model for the comparison of diagnostic tests. Am. J. Epidemiol. 83:593-602.

15. Georgiadis, M. P., Johnson, W. O., Gardner, I. A., and Singh, R. 2003. Correlation-adjusted estimation of sensitivity and specificity of two diagnostic tests. Appl. Stat. 52:63-76.

16. Gibbs, A., and Mackenzie, A. 1997. A primer pair for amplifying part of the genome of all potyvirids by RT-PCR. J. Virol. Methods 63:9-16.

17. Goodman, L. A. 1974. Exploratory latent structure analysis using both identifiable and unidentifiable models. Biometrika 61:215-231.

18. Goodman, L. A. 1974. The analysis of systems of qualitative variables when some of the variables are unobservable. Part I-A: Modified latent structure approach. Am. J. Sociol. 79:1179-1259.

19. Huber, M. 2006. Taking vital vines. Citrus Veg. Mag. 70:22-24

20. Hui, S. L., and Walter, S. D. 1980. Estimating the error rates of diagnostic tests. Biometrics 36:167-171.

21. Hui, S. L., and Zhou, X. H. 1998. Evaluation of diagnostic tests without gold standards. Stat. Med. Methods Res. 7:354-370.

22. Koukounari, A., Webster, J. P., Donnelly, C. A., Bray, B. C., Naples, J., Bosompen, K., and Shiff, C. 2009. Sensitivities and specificities of diagnostic tests and infection prevalence of Schistosoma haemaobium estimated from data on adults in villages northwest of Accra, Ghana. Am. J. Trop. Med. Hyg. 80:435-441.

23. Lanza S. T., Collins L. M., Lemmon, D. R., and Schafer, J. L. 2007. PROC LCA: A SAS procedure for latent class analysis. Struct. Equat. Model. 14:671-694.

24. Lanza, S. T., Dziak, J. J., Huang, L., Xu, S., and Collins, L. M. 2011. Proc LCA \& Proc LTA Users' Guide, Version 1.2.6. The Methodology Center, Pennsylvania State University, University Park. http://methodology. psu.edu

25. Lazarsfeld, P. F. 1950. The logical and mathematical foundations of latent structure analysis. In: Measurement and Prediction. S. A. Stouffer, L. Guttman, E. A. Suchman, P. F. Lazarsfeld, S. A. Star, and J. A. Clausen, eds. Princeton University Press, Princeton, NJ.

26. Ling, K.-S., Levi, A., Adkins, S., Kousik, C. S., Miller, G., Hassell, R., and Keinath, A. P. 2013. Development and field evaluation of multiple virus-resistant bottle gourd (Lagenaria siceraria). Plant Dis. 97:10571062.

27. McCutcheon, A. L. 1987. Latent Class Analysis. Sage University Paper Series on Quantitative Applications in the Social Sciences, Ser. No. 07064. SAGE Publications Inc., Newbury Park, CA.

28. Pouillot, R., Gerbier, G., and Gardner, I. A. 2002. "TAGS", a program for the evaluation of test accuracy in the absence of a gold standard. Prev. Vet. Med. 53:67-81.

29. Rindskopf, D., and Rindskopf, W. 1986. The value of latent class analysis in medical diagnosis. Stat. Med. 5:21-27.

30. Singer, R. S., Boyce, W. M., Gardner, I. A., Johnson, W. O., and Fisher, A. S. 1988. Evaluation of bluetongue virus diagnostic tests in free-ranging bighorn sheep. Prev. Vet. Med. 35:265-282.

31. Turechek, W. W., Irey, M., Sieburth, P., Brlansky, R., DaGraça, J., Graham, J., Gottwald, T., Hartung, J., Hilf, M., Kunta, M., Manjunath, K., Lin, H., Ramadugu, C., Roberts, P., Rogers, M., Shatters, R., Sun, X., and Wang, N. 2010. Evaluation of quantitative real-time PCR assays for detection of citrus greening. Pages 158-160 in: Proc. 10th Int. Epidemiol. Workshop, Geneva, NY.

32. Turechek, W. W., Kousik, C. S., and Adkins, S. 2010. Distribution of four viruses in single and mixed infection within infected watermelon plants in Florida. Phytopathology 100:1194-1203.

33. Vidal, E., Moreno, J., Bertolini, E., and Cambra, M. 2012. Estimation of the accuracy of two diagnostic methods for the detection of Plum pox virus in nursery blocks by latent class models. Plant Pathol. 61:413-422.

34. Walter, S. D., and Irwig, L. M. 1988. Estimation of test error rates, disease prevalence and relative risk from misclassified data: A review. J. Clin. Epidemiol. 41:923-937.

35. Webster C. G., and Adkins, S. 2012. Low genetic diversity of Squash vein yellowing virus in wild and cultivated cucurbits in the U.S. suggests a recent introduction. Virus Res. 163:520-527.

36. Webster, C. G., Kousik, C. S., Turechek, W. W., Webb, S. E., Roberts, P. D., and Adkins, S. 2013. Squash vein yellowing virus infection of vining cucurbits and the vine decline response. Plant Dis.97:1149-1157.

37. Yang, I., and Becker, M. P. 1997. Latent variable modeling of diagnostic accuracy. Biometrics 53:948-958. 